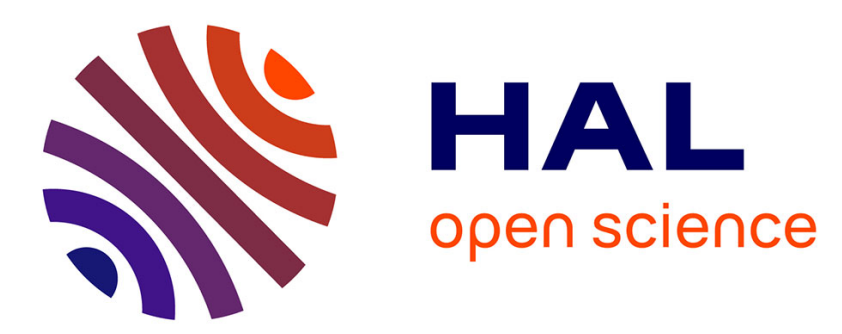

\title{
CoMP-Based Dynamic Handover for Vehicular VLC Networks
}

\author{
M. Selim Demir, Hossien B Eldeeb, Murat Uysal
}

\section{To cite this version:}

M. Selim Demir, Hossien B Eldeeb, Murat Uysal. CoMP-Based Dynamic Handover for Vehicular VLC Networks. IEEE Communications Letters, 2020, 24 (9), pp.2024-2028. 10.1109/LCOMM.2020.2994416 . hal-03341277

\section{HAL Id: hal-03341277 https://hal-amu.archives-ouvertes.fr/hal-03341277}

Submitted on 10 Sep 2021

HAL is a multi-disciplinary open access archive for the deposit and dissemination of scientific research documents, whether they are published or not. The documents may come from teaching and research institutions in France or abroad, or from public or private research centers.
L'archive ouverte pluridisciplinaire HAL, est destinée au dépôt et à la diffusion de documents scientifiques de niveau recherche, publiés ou non, émanant des établissements d'enseignement et de recherche français ou étrangers, des laboratoires publics ou privés. 


\title{
CoMP-Based Dynamic Handover for Vehicular VLC Networks
}

\author{
M. Selim Demir ${ }^{\circledR}$, Hossien B. Eldeeb ${ }^{\circledR}$, Student Member, IEEE, and Murat Uysal, Fellow, IEEE
}

\begin{abstract}
Visible light communication (VLC) has emerged as a potential wireless connectivity solution for infrastructure-tovehicle networks where street lights can be configured to serve as access points. In this letter, we propose dynamic soft handover algorithm based on coordinated multipoint (CoMP) transmission. The proposed algorithm takes the rate of change in the received power as an input and accordingly revises the handover margin and time-to-trigger value without explicit information of the vehicle velocity. Our simulation results demonstrate that the proposed algorithm outperforms conventional CoMP and hard handover and maintains a stable signal quality regardless of vehicle velocity.
\end{abstract}

Index Terms-Visible light communications, handover, vehicular network, CoMP.

\section{INTRODUCTION}

$\mathbf{V}$ EHICULAR networking is an essential component of intelligent transportation systems (ITS) and builds on the reliable and scalable implementation of vehicle-to-vehicle $(\mathrm{V} 2 \mathrm{~V})$, vehicle-to-infrastructure (V2I) and infrastructure-tovehicle (I2V) links [1], [2]. The current research activities and standardization efforts on vehicular networking mainly focus on radio frequency (RF) technologies [3], [4]. However, limited RF bands can quickly suffer from high interference levels when hundreds of vehicles located in the same vicinity try to communicate simultaneously. In such user-dense environments, channel congestion might result in longer delays and lower packet rates. To address such issues, visible light communication (VLC) has been proposed as an alternative means for vehicular connectivity [5]-[7].

VLC is based on the principle of modulating the intensity of light emitting diodes (LEDs) without impact on illumination levels or human eye. In the context of vehicular networking, automotive headlight, street and traffic lamps can be potentially used as VLC transmitters. In particular, uniformly placed highway street lights provide the required infrastructure for the implementation of $\mathrm{I} 2 \mathrm{~V}$ communication network where each of VLC-enabled street lights can be configured to serve as an access point (AP).

A critical issue in such a $\mathrm{I} 2 \mathrm{~V}$ network is the design of handover process required for efficient mobility management particularly given the relatively small coverage area of each light. Vertical and horizontal handover were studied

Manuscript received March 25, 2020; revised April 28, 2020; accepted May 10, 2020. The work of H. B. Eldeeb was supported by the Horizon 2020 MSC ITN (VISION) under Grant 764461. The work of M. Uysal was supported by the Turkish Scientific and Research Council (TUBITAK) under Grant 215E311. The associate editor coordinating the review of this letter and approving it for publication was C. Gong. (Corresponding author: M. Selim Demir.)

M. Selim Demir is with the Department of Electrical and Electronics Engineering, Özyeğin University, 34794 Istanbul, Turkey, and also with the TÜBITAK BILGEM, 41470 İzmit, Turkey (e-mail: mselimdemir@ gmail.com).

Hossien B. Eldeeb and Murat Uysal are with the Department of Electrical and Electronics Engineering, Özyeğin University, 34794 Istanbul, Turkey.

Digital Object Identifier 10.1109/LCOMM.2020.2994416

extensively in indoor VLC networks see e.g., [8]-[13] and references therein. Handover schemes proposed for indoor mobile VLC scenarios (i.e., optimized for pedestrian speeds) might be perhaps applicable for outdoor VLC systems if the vehicle velocity is sufficiently small. However, in general, the fast movement will seriously degrade the performance of such a system and make the system non-functional. This prompted researchers to investigate custom-design handover solutions for vehicular VLC networks [14]-[17].

The need for efficient handover in vehicular VLC networks was emphasized in [14], [15] without explicit details on the type of handover techniques. In [16], Dang and Yoo considered a I2V network where a number of consecutive street lights are grouped as a cell. Under the assumption of an on-board camera used as a receiver, they proposed an inter-cell handover technique. The handover is based on the estimated distance between the vehicle and each group of street lights and the required distance estimation is obtained through image processing techniques. In [17], N. Zhu et.al. considered a vehicular scenario where individual street lights serve as APs. They assumed both cases of overlapping and non-overlapping coverages and calculated received signal powers based on the Lambertian source model. Based on received signal strengths, they proposed a soft handover algorithm as a function of signal detection threshold and signal drop threshold. This handover scheme relies on the knowledge about the vehicle velocity and its implementation requires the estimation and feedback of velocity information.

In this letter, we propose dynamic soft handover algorithm based on coordinated multipoint (CoMP) transmission. CoMP transmission has been well investigated in the wireless communication literature and already standardized in the LTE-A [18]. The vehicle is mainly served by an AP from which it gets the strongest signal, but in the case of CoMP, the vehicle is jointly served by two coordinating APs. Based on the rate of change in the received powers (related to the vehicle velocity), the proposed handover algorithm dynamically revises the handover margin and time-to-trigger value in handover decision. In our algorithm, the handover margin typically increases while time-to-trigger value decreases for high-speed vehicles. This enables CoMP transmission to start early and maintain better signal quality. On the other hand, for low-speed vehicles, the handover margin is automatically set low and time-to-trigger value increases. This prevents the occurrence of ping-pong handovers ${ }^{1}$ and avoids unnecessary start of CoMP transmission in order to conserve the system resources. We evaluate the performance of proposed handover algorithm using realistic site-specific channel models developed through non-sequential ray tracing in OpticStudio ${ }^{\circledR}$ and

\footnotetext{
${ }^{1}$ Ping-pong handovers occur when the user is handed over from one cell to another but is quickly handed back to the original cell. This causes unnecessary signaling overhead and is an indication of incorrect handover parameter settings.
} 


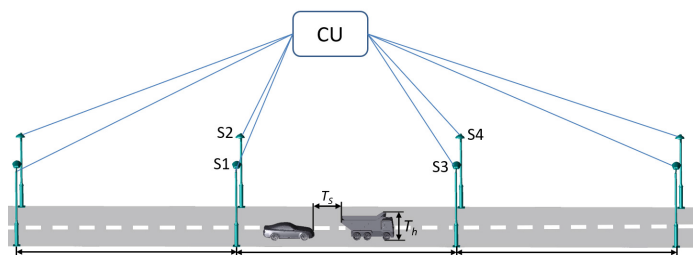

(a)

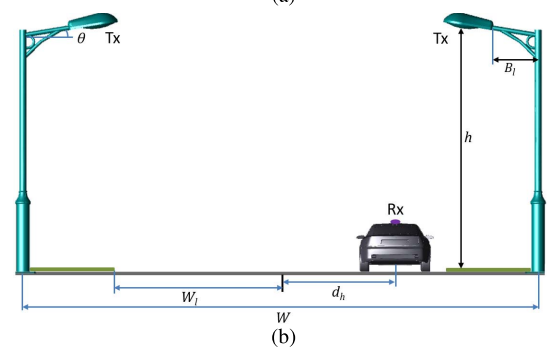

Fig. 1. (a) VLC-based I2V network (b) View from roadside.

demonstrate significant performance gains over conventional handover techniques.

The remainder of this letter is organized as follows. In Section II, we describe our system model. In Section III, we present the proposed dynamic handover algorithm. In Section IV, we present simulation results to demonstrate the performance of proposed algorithm. Finally, we conclude in Section V.

\section{SYSTEM MODEL}

As illustrated in Fig. 1.a, we consider a vehicular VLC network with multiple APs in the form of street light poles. The poles are uniformly arranged and separated from each other with a spacing of $L$. The centralized controller (CU) and APs are connected with wired connections and the CU acts as a gateway to Internet. As illustrated in Fig. 1.b, we assume a two-lane highway road with a lane width of $W_{l}$. Each of light poles has a height of $h$, boom length (distance between the center of the pole and the luminaire) of $B_{l}$, and boom angle of $\theta$. We assume that the receiver (vehicle) is located at a distance of $d_{h}$ (with respect to the road center) and travels with a velocity of $v$. It is equipped with a single photodetector located at the top of the car. It is possible that there might be other vehicles nearby, for example, a truck as illustrated in Fig. 1.a.

The physical layer of downlink builds upon direct current biased optical orthogonal frequency division multiplexing (DCO-OFDM). At the transmitter, the input bit stream is mapped into complex symbols, i.e., $\mathrm{s}_{1} \mathrm{~s}_{2} \cdots \mathrm{s}_{(K / 2)-1}$ where $K$ is the number of subcarriers. Hermitian symmetry is imposed on the data vector to ensure that the output of inverse discrete Fourier transform (IDFT) block is real. Therefore, the resulting data vector for the $i^{t h} \mathrm{AP}$ has the form of

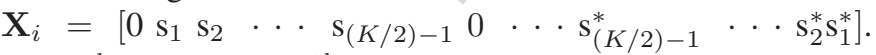
Let $X_{i}^{k}$ denote the $k^{t h}$ element of $\mathbf{X}_{i}$. Furthermore, let $P_{e}$ and $\rho$ respectively denote the electrical power and electricalto-optical conversion ratio. After $K$-point IDFT operation, the transmitted waveform from the $i^{\text {th }} \mathrm{AP}$ is written as [19]

$$
x_{i}(t)=\sum_{k=0}^{K-1} \frac{1}{\sqrt{K}} X_{i}^{k} e^{j \frac{2 \pi k}{K} t}+x_{D C} \quad t=0,1, \cdots, K-1
$$

where $x_{D C}=\rho \sqrt{P_{e}}$ is the DC bias. The average optical power is therefore given by $P_{\mathrm{opt}}=\mathbb{E}\left[x_{i}(t)\right]=x_{D C}$. It is also possible to write the relationship between electrical power and optical power as $P_{e}=P_{\mathrm{opt}}^{2} / \rho^{2}$ [19].

At the destination vehicle, the light intensity is detected by a photodetector. Let $H_{i}(t), i=1, \ldots, N_{A P}$, denote the DC channel gain from $i^{t h}$ AP to the vehicle. The received signal can be expressed as [19]

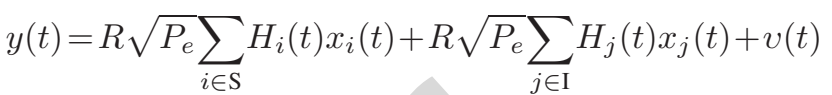

where $R$ is the photodetector responsivity. In the case of CoMP, the user is jointly served by coordinating APs that transmit the same information. Therefore, the set of $S$ includes two APs, otherwise it is limited to a single serving AP. $I$ denotes the set of interfering APs. In (2), $v(t)$ is the additive white Gaussian noise (AWGN) term with zero mean and variance of $\sigma^{2}=N_{0} B$. Here, $N_{0}$ is the noise power spectral density (PSD) and $B$ is the modulation bandwidth. Based on (2), the SINR at destination vehicle can be expressed as [20]

$$
\gamma(t)=\frac{\sum_{i \in \mathrm{S}} P_{i}(t)}{\sum_{j \in \mathrm{I}} P_{j}(t)+\sigma^{2}}
$$

where $P_{i}(t)=R^{2} P_{e} H_{i}^{2}(t)$ is the received electrical power from the $i^{t h} \mathrm{AP}$.

\section{CoMP BASEd DynAmic HANDOVER}

Based on the received power strengths, the $\mathrm{CU}$ decides which APs should serve the vehicle. If there is a sufficiently strong AP signal, the vehicle is served by that specific AP. In transition regions between two cells, the vehicle is jointly served by two coordinating APs as a result of CoMP transmission. Based on the rate of change in the received powers, the proposed handover algorithm dynamically revises the handover margin $(H O M)$ and time-to-trigger value $(T T T)$ in handover decision.

Selection of HOM and TTT values are critical for vehicular networks. TTT value should be low for high-speed vehicles because when a rapidly moving vehicle approaches the cell edge, the received signal from the serving AP drops rapidly and handover should be triggered immediately. On the other hand, for low-speed vehicles, TTT value should be sufficiently high in order to prevent ping-pong effect. High-speed vehicles experience short dwell time that might cause connection losses due to the high handover rate. In order to improve the connectivity reliability, HOM value for high-speed vehicles should be set high compared to low-speed vehicles with relatively long cell dwell times. Unlike conventional CoMP where fixed values of $H O M$ and TTT are assumed, our proposed algorithm dynamically changes these threshold parameters.

The pseudo-code of the proposed handover algorithm is provided in Algorithm 1. Let $P_{c}(t)$ and $P_{s}(t)$ denote the received power from the candidate AP and the serving AP at time $t$, respectively. Furthermore, let $P_{s}(t-\Delta t)$ represent the received power from the serving AP at time $t-\Delta t$. The rate of the change in the received power is expressed as $\Delta P=$ $\left[P_{s}(t)-P_{s}(t-\Delta t)\right] / \Delta t$. Based on the value of $\Delta P, H O M$ and TTT are respectively calculated as $H O M=\alpha \Delta P$ and $T T T=\lambda / \Delta P$, where $\alpha$ and $\lambda$ are some constant coefficients. 


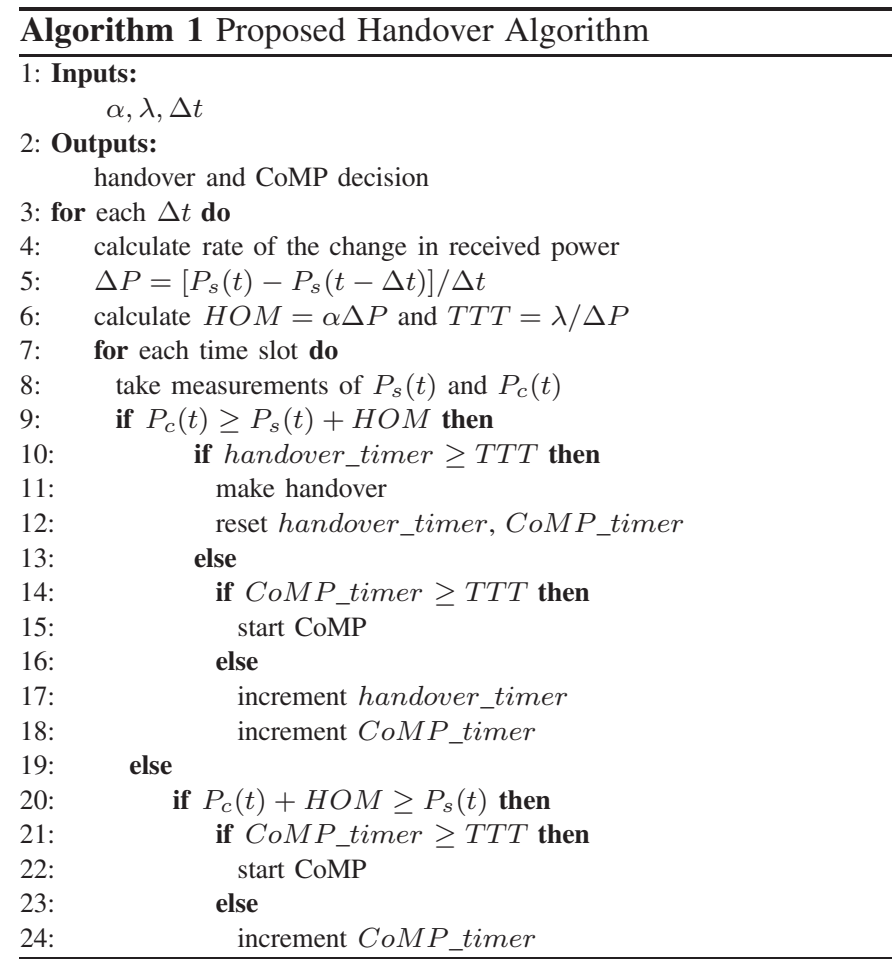

When a vehicle moves away from the serving AP, the received power of the serving AP decreases and the received power from the candidate AP increases. When the vehicle enters the region of the candidate AP and the condition $P_{c}(t)+H O M \geq$ $P_{s}(t)$ is satisfied for a certain time, CoMP transmission starts and the vehicle is jointly served by both serving and candidate APs. In CoMP phase, both APs transmit the same information and the received signals are combined at the receiver. Handover to the candidate AP is triggered when the $P_{c}(t) \geq P_{s}(t)+H O M$ is satisfied for a duration of TTT. The vehicle terminates its connection with the previous serving AP and continues getting service from the candidate AP. Therefore, candidate AP becomes the new serving AP and serves the vehicle alone until the new handover decision is taken.

A critical issue in the practical implementation is the choice of values of $\alpha$ and $\lambda$. TTT is a monotonically increasing function of $\lambda$ and a lower value of $\lambda$ is preferred which expedites the start of handover and CoMP transmission, but the value of $\lambda$ should be greater than 0 to prevent pingpong handovers. On the other hand, $H O M$ is a monotonically increasing function of $\alpha$. The choice of too small $\alpha$ would disable the CoMP transmission while a large choice of $\alpha$ would trigger unnecessary CoMP transmissions. As a rule of thumb, $\alpha$ and $\lambda$ values should be selected such that SINR remains almost constant regardless of the vehicle speed.

\section{Simulation Results}

In our simulations, we consider a two-lane road where the poles in the same lane are separated with a spacing of $L=20 \mathrm{~m}$, and each of them has a height of $h=7 \mathrm{~m}$. We consider two main use cases: Scenario A) The car travels in the right lane without any neighbor vehicles, Scenario B) The car travels in the right lane and precedes a loaded truck with a height of $T_{h}=4.2 \mathrm{~m}$. The distance between

TABLE I

Simulation PARAMETERS

\begin{tabular}{|l|l|l|}
\hline \multirow{3}{*}{ Transmitter } & Brand & Vestel Ephesus M3S 90 \\
& Optical power & $1 \mathrm{~W}$ \\
& Power ratio $(\eta)$ & 3 \\
\hline \multirow{3}{*}{ Receiver } & Area & $150 \mathrm{~mm}^{2}$ \\
& FOV & $180^{\circ}$ \\
& Responsivity $(R)$ & $1(\mathrm{~A} / \mathrm{W})$ \\
\hline \multirow{2}{*}{ Road } & Lane width $\left(W_{l}\right)$ & $3.75 \mathrm{~m}$ \\
& Road width $(W)$ & $10.5 \mathrm{~m}$ \\
\hline \multirow{2}{*}{ Car } & Dimensions & $4.67 \mathrm{~m} \times 1.85 \mathrm{~m} \times 1.37 \mathrm{~m}$ \\
& Material & Black gloss paint \\
\hline \multirow{2}{*}{ Truck } & Dimensions & $5.4 \mathrm{~m} \times 1.8 \mathrm{~m} \times 4.2 \mathrm{~m}$ \\
& Material & Black gloss paint \\
\hline \multirow{5}{*}{ Poles } & Material & Galvanized steel metal \\
& Spacing $(L)$ & $20 \mathrm{~m}$ \\
& Height $(h)$ & $7 \mathrm{~m}$ \\
& Boom Length $\left(B_{l}\right)$ & $1 \mathrm{~m}$ \\
& Boom Angle $(\theta)$ & $10^{\circ}$ \\
\hline \multirow{2}{*}{ Noise } & Noise density $\left(N_{0}\right)$ & $1 \times 10^{-20}\left(\mathrm{~A}^{2} / \mathrm{Hz}\right)$ \\
& Bandwidth $(\mathrm{B})$ & $20 \mathrm{MHz}$ \\
\hline
\end{tabular}

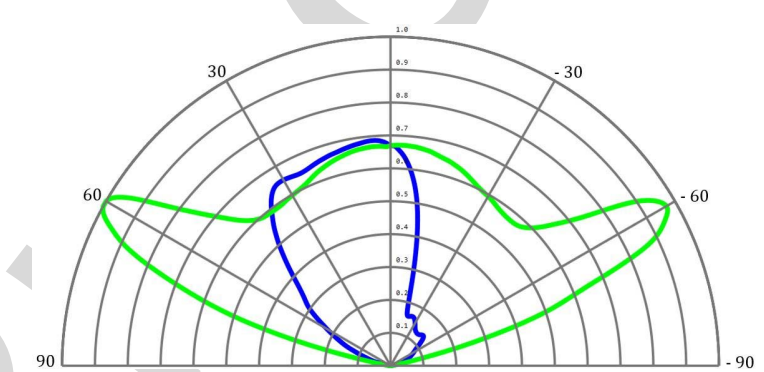

Fig. 2. Radiation pattern of street light under consideration; The green curve indicates the horizontal radiation pattern while blue curve represents the vertical one.

two vehicles is given by $T_{s}=4 \mathrm{~m}$. The second scenario is particularly useful to analyze the effect of potential blockage. The car is equipped with a single photodetector located at the top of the car (See Fig. 1b). It has an aperture area of $10 \mathrm{~mm} \times 15 \mathrm{~mm}$ and field-of-view (FOV) angle of $180^{\circ}$. $^{2}$ All simulation parameters are provided in Table I.

For channel modeling, we use the non-sequential ray tracing approach in [21]. We consider a commercial streetlamp with an asymmetrical radiation pattern as shown in Fig. 2 [22]. This pattern features a narrow vertical beam angle combined with a wide horizontal beam one. The benefit of the horizontal wide beam angle is to spread the light to longer distances along the road while the vertical narrow beam is required in order to focus the light to the road surfaces only. The LED radiation pattern is integrated into the three dimensional simulation environment constructed in OpticStudio ${ }^{\circledR}$ software. Channel impulse responses (CIRs) between each street light and destination vehicle are obtained based on non-sequential ray tracing features of this software at each 1 meter over the traveling distance between two poles. Based on earlier discussions in Section III, constant coefficients $\alpha$ and $\lambda$ are set as 200 and 0.1 , respectively.

In Fig. 3, we consider Scenario A (i.e., no blockage case) and assume that the car travels in the middle of the right lane (i.e., $d_{h}=2 \mathrm{~m}$ ). We present the average $H O M$ and

${ }^{2}$ A lower value of FOV will reduce the ambient noise. However, in an interference-limited case, this reduction will be negligible. Therefore, we preferred a wide FOV angle to maximize the reception angle in mobile conditions under consideration. 


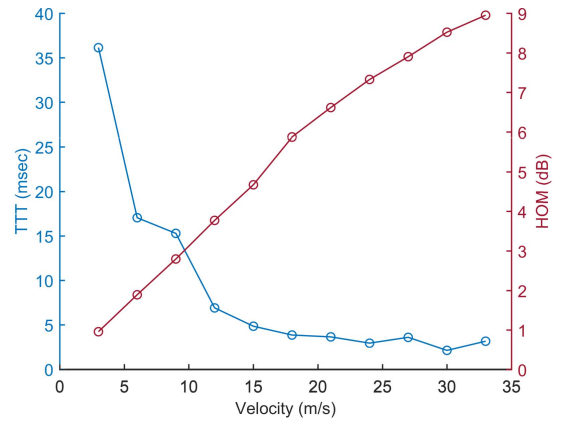

Fig. 3. HOM and TTT versus velocity.

$T T T$ values as a function of the vehicle speed to better highlight the necessity of dynamically revising these parameters which is the main feature of the proposed algorithm. It is observed that $T T T$ decreases with velocity. Since, the received signal drops rapidly for high-speed vehicles when they move away from the source, AP handover is initiated more rapidly for them in comparison to slower vehicles. On the other hand, HOM increases with speed. For high-speed vehicles, CoMP transmission starts earlier and continues longer. This makes system more reliable to sudden connection drops due to high velocity. Consequently, there is a combination of proper $H O M$ and TTT values that needs to be selected for each speed of the vehicle.

In Fig. 4, we present the performance of proposed handover algorithm for Scenario A. We assume that the vehicle travels at the center of lane with the speed of $18 \mathrm{~m} / \mathrm{s}(64.8 \mathrm{~km} / \mathrm{hr})$. As benchmarks, we consider four schemes: 1) Hard handover as specified in 3GPP document [23], 2) Best Connection (BC) algorithm [24] where the vehicle is always connected to the AP providing the received signal with the highest power, 3) Conventional CoMP handover where the user can be jointly served by two coordinating APs, 4) CoMP-Joint Processing (CoMP-JP) handover [25] which uses the average power of the received signals from the coordinated APs instead of the power of the received signal from the source AP (allowing the postponement of the handover if necessary).

In hard handover, handover margin is set as $H O M=1 \mathrm{~dB}$ while time-to-trigger is set as $T T T=160 \mathrm{~ms}$ in order to prevent ping-pong handovers [13]. In conventional CoMP, fixed parameters of $H O M=3 \mathrm{~dB}$ value and $T T T=80 \mathrm{~ms}$ value are assumed. ${ }^{3}$ The same values are also employed in CoMP-JP. ${ }^{4}$ In BC algorithm, in order to connect to the AP providing the received signal with highest power, $H O M=$ $0 \mathrm{~dB}$ and $T T T=0 \mathrm{~ms}$ are chosen as default. In the proposed algorithm, it can be readily checked from Fig. 3 that $H O M$ and $T T T$ values should be chosen respectively as $H O M=5.90 \mathrm{~dB}$ and $T T T=3.85 \mathrm{~ms}$ for the speed of $18 \mathrm{~m} / \mathrm{s}$ under consideration.

${ }^{3}$ Fixed values of HOM and TTT are used in conventional CoMP algorithm. Therefore, it is important to choose values which will provide a decent performance independent of the vehicle speed. In order to determine proper values, we simulated the performance of conventional CoMP for different $H O M$ and TTT values assuming vehicle speeds of $9 \mathrm{~m} / \mathrm{s}, 18 \mathrm{~m} / \mathrm{s}$ and $27 \mathrm{~m} / \mathrm{s}$. Based on these extensive simulations, we selected $H O M=3 \mathrm{~dB}$ and $T T T=80 \mathrm{~ms}$ which maintain a relatively stable SINR and fit better for all speeds.

${ }^{4}$ It is reported in [25] that CoMP-JP provides a superior performance for $2 \mathrm{~dB}<H O M<4 \mathrm{~dB}$.

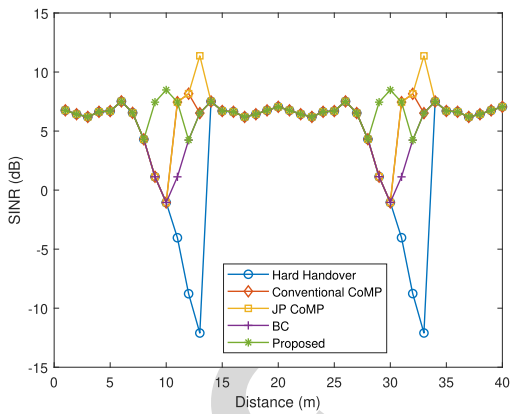

Fig. 4. Performance comparison of proposed handover algorithm with different techniques. Vehicle travels at the center of the lane with a speed of $18 \mathrm{~m} / \mathrm{s}$.

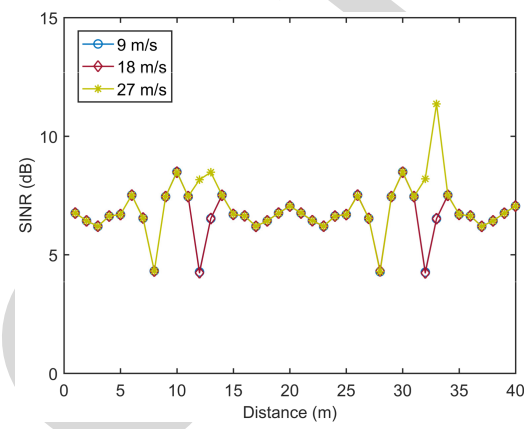

Fig. 5. SINR versus distance for proposed handover technique with different vehicle speeds.

It is observed from Fig. 4 that hard handover, conventional CoMP, CoMP-JP and $\mathrm{BC}$ algorithms have severe fluctuations in SINR. In hard handover, SINR drops as low as $-12.1 \mathrm{~dB}$ while lowest values experienced in conventional CoMP, CoMP-JP and BC handover schemes are significantly larger. CoMP-JP algorithm has similar performance to conventional CoMP, but at cell edges it triggers unnecessary CoMP transmission and causes unnecessary usage of system resources. On the other hand, as a result of the proper selection of $H O M$ and TTT values, the proposed handover technique maintains a more stable SINR and outperforms its counterparts. The lowest SINR value is obtained as $4.23 \mathrm{~dB}$ which is much higher than those in benchmarking schemes and therefore enables a better signal quality.

In Fig. 5, we investigate the effect of vehicle speed on the performance of proposed handover algorithm assuming Scenario A. We consider three different speeds: $9 \mathrm{~m} / \mathrm{s}$ $(32.4 \mathrm{~km} / \mathrm{hr}), 18 \mathrm{~m} / \mathrm{s}(64.8 \mathrm{~km} / \mathrm{hr})$ and $27 \mathrm{~m} / \mathrm{s}(97.2 \mathrm{~km} / \mathrm{hr})$. It is assumed that the vehicle travels at the center of lane. $H O M$ and TTT values are selected as $\{H O M=2.80$, $T T T=15.28\}$ for $9 \mathrm{~m} / \mathrm{s},\{H O M=5.90, T T T=3.85\}$ for $18 \mathrm{~m} / \mathrm{s},\{H O M=7.89, T T T=3.60\}$ for $27 \mathrm{~m} / \mathrm{s}$. It is observed that the proposed algorithm is able to maintain a stable SINR performance regardless of the vehicle speed. The average SINR values for $9 \mathrm{~m} / \mathrm{s}, 18 \mathrm{~m} / \mathrm{s}$ and $27 \mathrm{~m} / \mathrm{s}$ can be calculated respectively as $6.63 \mathrm{~dB}, 6.63 \mathrm{~dB}$ and $6.99 \mathrm{~dB}$. Similarly, the lowest SINR value experienced for different speeds remains around $4.25 \mathrm{~dB}$. These results demonstrate the performance stability of proposed algorithm for different speeds.

In Fig. 6, we investigate the effect of blockage on the proposed handover algorithm. The blue plot is obtained for Scenario A (i.e., no blockage) while the orange plot is obtained 


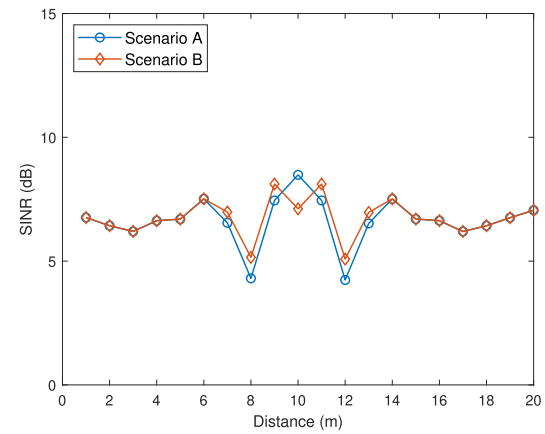

Fig. 6. Effect of blockage on the performance of proposed handover algorithm.

for Scenario B (i.e., with blockage). It is observed that blockage introduces some degradation around $d=10 \mathrm{~m}$ which corresponds to midway between two APs. Because at the cell edge, the vehicle is jointly served by $\mathrm{S} 1$ and $\mathrm{S} 3$ and, since the received power from S3 in Scenario B is lower than that received in Scenario A due to blockage, some degradation in SINR value is observed. Other than the cell edge, SINR values for blockage and non-blockage scenarios remain nearly the same in general. Interestingly, between $6 \mathrm{~m}<d<10 \mathrm{~m}$ and $10 \mathrm{~m}<d<14 \mathrm{~m}$, SINR values in blockage case are even slightly higher than those in non-blockage case. For $6 \mathrm{~m}<d<$ $10 \mathrm{~m}$, the car is served by $\mathrm{S} 1$ located behind the truck and the blockage reduces the interfering signal that comes from S3. Therefore, the blockage becomes beneficial in this case since it partially obstructs the interfering signal. Similarly, for $10 \mathrm{~m}<d<14 \mathrm{~m}$, the car is served by S3 while the received power from S4 (interfering signal) is reduced due to the blockage effect.

\section{CONCLUSION}

In this letter, we have proposed a CoMP based dynamic handover algorithm for vehicular VLC networks. Unlike the conventional CoMP, the proposed algorithm dynamically revises the HOM and TTT values based on the rate of change in the received power. Our simulation results show that $T T T$ should take small values for high-speed vehicles while it increases for low-speed vehicles in order to prevent ping-pong effect. On the contrary, HOM should take high values for highspeed vehicles while it should decrease for low-speed vehicles with relatively long cell dwell times. With proper selection of HOM and TTT values, our proposed algorithm outperforms the conventional CoMP and hard handover and maintains higher and more stable SINR performance. Our simulation results further reveal that the proposed algorithm provides a stable performance even in the presence of nearby vehicles which can result in partial blockage of the received signal.

\section{REFERENCES}

[1] K. Zheng, Q. Zheng, P. Chatzimisios, W. Xiang, and Y. Zhou, "Heterogeneous vehicular networking: A survey on architecture, challenges, and solutions," IEEE Commun. Surveys Tuts., vol. 17, no. 4, pp. 2377-2396, 4th Quart., 2015.

[2] J. Guo, B. Song, Y. He, F. R. Yu, and M. Sookhak, "A survey on compressed sensing in vehicular infotainment systems," IEEE Commun. Surveys Tuts., vol. 19, no. 4, pp. 2662-2680, 4th Quart., 2017.
[3] S. Chen, J. Hu, Y. Shi, and L. Zhao, "LTE-V: A TD-LTE-based V2X solution for future vehicular network," IEEE Internet Things J., vol. 3 , no. 6, pp. 997-1005, Dec. 2016.

[4] K. Abboud, H. A. Omar, and W. Zhuang, "Interworking of DSRC and cellular network technologies for V2X communications: A survey," IEEE Trans. Veh. Technol., vol. 65, no. 12, pp. 9457-9470, Dec. 2016.

[5] M. Uysal, Z. Ghassemlooy, A. Bekkali, A. Kadri, and H. Menouar, "Visible light communication for vehicular networking: Performance study of a V2 V system using a measured headlamp beam pattern model," IEEE Veh. Technol. Mag., vol. 10, no. 4, pp. 45-53, Dec. 2015.

[6] J.-H. Yoo, J.-S. Jang, J. K. Kwon, H.-C. Kim, D.-W. Song, and S.-Y. Jung, "Demonstration of vehicular visible light communication based on LED headlamp." Int. J. Automot. Technol., vol. 17, no. 2, pp. 347-352, Apr. 2016.

[7] A.-M. Cailean and M. Dimian, "Current challenges for visible light communications usage in vehicle applications: A survey," IEEE Commun. Surveys Tuts., vol. 19, no. 4, pp. 2681-2703, 4th Quart., 2017.

[8] F. Wang, Z. Wang, C. Qian, L. Dai, and Z. Yang, "Efficient vertical handover scheme for heterogeneous VLC-RF systems," J. Opt. Commun. Netw., vol. 7, no. 12, pp. 1172-1180, Dec. 2015.

[9] S. Liang, Y. Zhang, B. Fan, and H. Tian, "Multi-attribute vertical handover decision-making algorithm in a hybrid VLC-Femto system," IEEE Commun. Lett., vol. 21, no. 7, pp. 1521-1524, Jul. 2017.

[10] E. Dinc, O. Ergul, and O. B. Akan, "Soft handover in OFDMA based visible light communication networks," in Proc. IEEE 82nd Veh. Technol. Conf. (VTC-Fall), Sep. 2015, pp. 1-5.

[11] M. D. Soltani, H. Kazemi, M. Safari, and H. Haas, "Handover modeling for indoor Li-Fi cellular networks: The effects of receiver mobility and rotation," in Proc. IEEE Wireless Commun. Netw. Conf. (WCNC), Mar. 2017, pp. 1-6.

[12] M. Hammouda, J. Peissig, and A. M. Vegni, "Design of a cognitive VLC network with illumination and handover requirements," in Proc. IEEE Int. Conf. Commun. Workshops (ICC Workshops), May 2017, pp. $451-456$.

[13] X. Wu and H. Haas, "Handover skipping for LiFi," IEEE Access, vol. 7, pp. 38369-38378, 2019.

[14] P. Arunachalam and N. Kumar, "Visible light communication and radio network for vehicular environment," in Proc. 2nd Int. Conf. Adv Electron., Comput. Commun. (ICAECC), Feb. 2018, pp. 1-5.

[15] E. Torres-Zapata, V. Guerra, J. Rabadan, R. Perez-Jimenez, and J. M. Luna-Rivera, "Vehicular communications in tunnels using VLC," in Proc. 15th Int. Conf. Telecommun. (ConTEL), Jul. 2019, pp. 1-6.

[16] Q.-H. Dang and M. Yoo, "Handover procedure and algorithm in vehicle to infrastructure visible light communication," IEEE Access, vol. 5, pp. 26466-26475, 2017.

[17] N. Zhu, Z. Xu, Y. Wang, H. Zhuge, and J. Li, "Handover method in visible light communication between the moving vehicle and multiple LED streetlights," Optik-Int. J. Light Electron Opt., vol. 125, no. 14, pp. 3540-3544, Jul. 2014.

[18] 3rd Generation Partnership Project; Technical Specification Group Radio Access Network; Feasibility study for Further Advancements for E-UTRA (LTE-Advanced) (Release 10), V10.0.0, document TR36.912, 3GPP, 2011.

[19] H. Kazemi and H. Haas, "Downlink cooperation with fractional frequency reuse in DCO-OFDMA optical attocell networks," in Proc. IEEE Int. Conf. Commun. (ICC), May 2016, pp. 1-6, doi: 10.1109/icc.2016. 7511475.

[20] Y. Wang and H. Haas, "Dynamic load balancing with handover in hybrid Li-Fi and Wi-Fi networks," J. Lightw. Technol., vol. 33, no. 22, pp. 4671-4682, Nov. 15, 2015.

[21] M. Elamassie, M. Karbalayghareh, F. Miramirkhani, R. C. Kizilirmak, and M. Uysal, "Effect of fog and rain on the performance of vehicular visible light communications," in Proc. IEEE 87th Veh. Technol. Conf (VTC Spring), Jun. 2018, pp. 1-6.

[22] Vestel Emphesis. [Online]. Available: http://www.vestelledlighting.com

[23] Evolved Universal Terrestrial Radio Access (E-UTRA); Radio Resource Control (RRC); Protocol Specification, Version 14.2.2, document 36.331, 3GPP, Apr. 2017.

[24] K. Da Costa Silva, Z. Becvar, and C. R. L. Frances, "Adaptive hysteresis margin based on fuzzy logic for handover in mobile networks with dense small cells," IEEE Access, vol. 6, pp. 17178-17189, 2018.

[25] A. Nakano and T. Saba, "A handover scheme based on signal power of coordinated base stations for CoMP joint processing systems," in Proc. 8th Int. Conf. Signal Process. Commun. Syst. (ICSPCS), Dec. 2014, pp. 1-6. 